\title{
On Certain Subclasses of Multivalent Functions Associated with a Family of Linear Operators
}

\author{
Jae Ho Choi \\ Department of Mathematics Education, Daegu National University of Education, Daegu, South Korea \\ E-mail:choijh@dnue.ac.kr \\ Received March 16, 2011; revised April 7, 2011; accepted April 20, 2011
}

\begin{abstract}
Making use of a linear operator $\mathcal{I}_{p}^{\lambda}(a, c)$, which is defined here by means of the Hadamard product (or convolution), we introduce some new subclasses of multivalent functions and investigate various inclusion properties of these subclasses. Some radius problems are also discussed.
\end{abstract}

Keywords: Multivalent Functions, Hadamard Product (or Convolution), Linear Operators, Radius Problem

\section{Introduction and Definitions}

Let $\mathcal{A}(p)$ denote the class of functions $f(z)$ of the form

$$
f(z)=z^{p}+\sum_{k=1}^{\infty} a_{p+k} z^{p+k} \quad(p \in \mathbb{N}:=\{1,2,3, \cdots\}),
$$

which are analytic in the open unit disk

$$
\mathbb{U}=\{z: z \in \mathbb{C} \text { and }|z|<1\} .
$$

We define the Hadamard product (or convolution) of two analytic functions

$$
f(z)=\sum_{k=0}^{\infty} a_{k} z^{k} \text { and } g(z)=\sum_{k=0}^{\infty} b_{k} z^{k},
$$

as

$$
(f * g)(z):=\sum_{k=0}^{\infty} a_{k} b_{k} z^{k} \quad(z \in \mathbb{U}) .
$$

For $a \in \mathbb{R}, c \in \mathbb{R} / \mathbb{Z}_{0}^{-}\left(\mathbb{Z}_{0}^{-}:=\{\cdots,-2,-1,0\}\right)$ H. Saitoh [13] introduced a linear operator

$$
\mathcal{L}_{p}(a, c): \mathcal{A}(p) \rightarrow \mathcal{A}(p)
$$

defined by

$$
\mathcal{L}_{p}(a, c) f(z):=\phi_{p}(a, c ; z) * f(z) \quad(z \in \mathbb{U} ; f \in \mathcal{A}(p))
$$

where

$$
\phi_{p}(a, c ; z):=\sum_{k=0}^{\infty} \frac{(a)_{k}}{(c)_{k}} z^{k+p} \quad(z \in \mathbb{U})
$$

and $(\tau)_{k}$ is the Pochhammer symbol defined, in terms of the Gamma function, by

$$
(\tau)_{k}=\frac{\Gamma(\tau+k)}{\Gamma(\tau)}= \begin{cases}1 & (k=0) \\ \tau(\tau+1) \cdots(\tau+k-1) & (k \in \mathbb{N}) .\end{cases}
$$

The operator $\mathcal{L}_{p}(a, c)$ is an extension of the CarlsonShaffer operator (see [2]). In [3], Cho et al. introduced the following family of linear operators

$\mathcal{I}_{p}^{\lambda}(a, c): \mathcal{A}(p) \rightarrow \mathcal{A}(p)$ analogous to $\mathcal{L}_{p}(a, c)$ (see also [14]):

$$
\begin{aligned}
& \mathcal{I}_{p}^{\lambda}(a, c) f(z):=\phi_{p}^{\dagger}(a, c ; z) * f(z) \\
& \left(a, c \in \mathbb{R} / \mathbb{Z}_{0}^{-} ; \lambda>-p ; z \in \mathbb{U} ; f \in \mathcal{A}(p)\right) .
\end{aligned}
$$

where $\phi_{p}^{\dagger}(a, c ; z)$ is the function defined in terms of the Hadamard product (or convolution) by the following condition

$$
\phi_{p}(a, c ; z) * \phi_{p}^{\dagger}(a, c ; z)=\frac{z^{p}}{(1-z)^{\lambda+p}},
$$

where $\phi_{p}$ is given by (3). If $f(z)$ is given by (1), then from (3), (4) and (5), we deduce that

$$
\begin{aligned}
& \mathcal{I}_{p}^{\lambda}(a, c) f(z) \\
& =z^{p}+\sum_{k=1}^{\infty} \frac{(\lambda+p)_{k}(c)_{k}}{(a)_{k} k !} a_{k+p} z^{k+p} \quad(z \in \mathbb{U}) .
\end{aligned}
$$

It is easily seen from (6) that

$\mathcal{I}_{p}^{1}(p+1,1) f(z)=f(z)$ and $\mathcal{I}_{p}^{1}(p, 1) f(z)=\frac{z f^{\prime}(z)}{p}$ 


$$
\begin{aligned}
& z\left(\mathcal{I}_{p}^{\lambda}(a+1, c) f(z)\right)^{\prime} \\
& =a \mathcal{I}_{p}^{\lambda}(a, c) f(z)-(a-p) \mathcal{I}_{p}^{\lambda}(a+1, c) f(z)
\end{aligned}
$$

and

$$
\begin{aligned}
& z\left(\mathcal{I}_{p}^{\lambda}(a, c) f(z)\right)^{\prime} \\
& =(\lambda+p) \mathcal{I}_{p}^{\lambda+1}(a, c) f(z)-\lambda \mathcal{I}_{p}^{\lambda}(a, c) f(z) .
\end{aligned}
$$

Clearly, from (7) and (8), we have

$$
\begin{aligned}
& \operatorname{Re}\left(\frac{z\left(\mathcal{I}_{p}^{\lambda}(a+1, c) f(z)\right)^{\prime}}{\mathcal{I}_{p}^{\lambda}(a+1, c) f(z)}\right)>0 \\
& \Leftrightarrow \operatorname{Re}\left(\frac{\mathcal{I}_{p}^{\lambda}(a, c) f(z)}{\mathcal{I}_{p}^{\lambda}(a+1, c) f(z)}\right)>\frac{a-p}{a} \quad(a \geq p)
\end{aligned}
$$

and

$$
\begin{aligned}
& \operatorname{Re}\left(\frac{z\left(\mathcal{I}_{p}^{\lambda}(a+1, c) f(z)\right)^{\prime}}{\mathcal{I}_{p}^{\lambda}(a, c) f(z)}\right)>0 \\
& \Leftrightarrow \operatorname{Re}\left(\frac{\mathcal{I}_{p}^{\lambda+1}(a, c) f(z)}{\mathcal{I}_{p}^{\lambda}(a, c) f(z)}\right)>\frac{\lambda}{\lambda+p} \quad(\lambda \geq 0) .
\end{aligned}
$$

When $a=n+p\left(n \in \mathbb{N}_{0}:=\mathbb{N} \cup\{0\}\right)$ and $c=\lambda=1$, the linear operator $\mathcal{I}_{p}^{1}(n+p, 1)=\mathcal{I}_{n+p-1}$, was introduced and studied by Liu and Noor [5] (see also [9] and [10]). Moreover, when $p=1, \mathcal{I}_{1}^{1}(n+1,1)=\mathcal{I}_{n}$ was first introduced and studied by Noor [8] which is known as Noor Integral operator.

Let $\mathcal{P}_{k}(\alpha)$ be the class of functions $h(z)$ analytic in the unit disk $\mathbb{U}$ satisfying the properties $h(0)=1$ and

$$
\int_{0}^{2 \pi}\left|\frac{\operatorname{Re} h(z)-\alpha}{1-\alpha}\right| \mathrm{d} \theta \leq k \pi,
$$

where $z=r e^{i \theta}, k \geq 2$ and $0 \leq \alpha<1$. For $\alpha=0$, the class $\mathcal{P}_{k}(0)=\mathcal{P}_{k}$ was introduced in [11]. For $\alpha=0$, $k=2$, we have the well known class $\mathcal{P}$ of functions with $\operatorname{Re} h(z)>0$ and the class $k=2$ gives us the class $\mathcal{P}(\alpha)$ of functions with $\operatorname{Re} h(z)>\alpha$. Also we can write, for $h(z) \in \mathcal{P}_{k}(\alpha)$ as

$$
h(z)=\frac{1}{2} \int_{0}^{2 \pi} \frac{1+(1-2 \alpha) z e^{-i t}}{1-z e^{-i t}} \mathrm{~d} \mu(t),
$$

where $\mu(t)$ is a function with bounded variation on $[0,2 \pi]$ such that

$$
\int_{0}^{2 \pi} \mathrm{d} \mu(t)=2 \quad \text { and } \quad \int_{0}^{2 \pi}|\mathrm{d} \mu(t)| \leq k .
$$

From (11) and (12) it can be seen that $h \in \mathcal{P}_{k}(\alpha)$ if and only if there exist $h_{1}, h_{2} \in \mathcal{P}(\alpha)$ such that

$$
h(z)=\left(\frac{k}{4}+\frac{1}{2}\right) h_{1}(z)-\left(\frac{k}{4}-\frac{1}{2}\right) h_{2}(z) .
$$

It is known [7] that the class $\mathcal{P}_{k}(\alpha)$ is a convex set. We also note that $h(z) \in \mathcal{P}_{k}(\alpha)$ if and only if there exists $q \in \mathcal{P}_{k}$ such that

$$
h(z)=(1-\alpha) q(z)+\alpha .
$$

By using the linear operator $\mathcal{I}_{p}^{\lambda}(a, c)$, we now define some subclasses of $\mathcal{A}(p)$ as follows:

Definition 1: Let $a \geq p, c \in \mathbb{R} / \mathbb{Z}_{0}^{-}, \lambda>-p, \mu>0$, $\eta \geq 0, k \geq 2$ and $0 \leq \alpha<1$. A function $f(z) \in \mathcal{A}(p)$ is said to be in the class $\mathcal{H}_{p}^{\lambda}(k, a, c, \alpha, \beta, \mu, \eta)$ if and only if it satisfies

$$
\begin{aligned}
& \left\{(1-\eta)\left(\frac{\mathcal{I}_{p}^{\lambda}(a+1, c) f(z)}{\mathcal{I}_{p}^{\lambda}(a+1, c) g(z)}\right)^{\mu}\right. \\
& \left.+\eta\left(\frac{\mathcal{I}_{p}^{\lambda}(a, c) f(z)}{\mathcal{I}_{p}^{\lambda}(a, c) g(z)}\right)\left(\frac{\mathcal{I}_{p}^{\lambda}(a+1, c) f(z)}{\mathcal{I}_{p}^{\lambda}(a+1, c) g(z)}\right)^{\mu-1}\right\} \in \mathcal{P}_{k}(\alpha),
\end{aligned}
$$

where $g(z) \in \mathcal{A}(p)$ satisfies the condition

$$
\left\{\frac{\mathcal{I}_{p}^{\lambda}(a, c) g(z)}{\mathcal{I}_{p}^{\lambda}(a+1, c) g(z)}\right\} \in \mathcal{P}(\beta) \quad(0 \leq \beta<1 ; z \in \mathbb{U}) .
$$

We note that $g$ is starlike univalent in $\mathbb{U}$ when $a=c=p=\lambda=1$ in (16).

Definition 2: Let $a, c \in \mathbb{R} / \mathbb{Z}_{0}^{-}, \lambda \geq 0, \mu>0, \eta \geq 0$, $k \geq 2$ and $0 \leq \alpha<1$. A function $f(z) \in \mathcal{A}(p)$ is said to be in the class $\mathcal{Q}_{p}^{\lambda}(k, a, c, \alpha, \beta, \mu, \eta)$ if and only if it satisfies

$$
\begin{aligned}
& \left\{(1-\eta)\left(\frac{\mathcal{I}_{p}^{\lambda}(a, c) f(z)}{\mathcal{I}_{p}^{\lambda}(a, c) g(z)}\right)^{\mu}\right. \\
& \left.+\eta\left(\frac{\mathcal{I}_{p}^{\lambda+1}(a, c) f(z)}{\mathcal{I}_{p}^{\lambda+1}(a, c) g(z)}\right)\left(\frac{\mathcal{I}_{p}^{\lambda}(a, c) f(z)}{\mathcal{I}_{p}^{\lambda}(a, c) g(z)}\right)^{\mu-1}\right\} \in \mathcal{P}_{k}(\alpha),
\end{aligned}
$$

where $g(z) \in \mathcal{A}(p)$ satisfies the condition

$$
\left\{\frac{\mathcal{I}_{p}^{\lambda+1}(a, c) f(z)}{\mathcal{I}_{p}^{\lambda}(a, c) g(z)}\right\} \in \mathcal{P}(\beta) \quad(0 \leq \beta<1 ; z \in \mathbb{U}) .
$$

In this manuscript, we investigate several inclusion and other properties of functions in the classes $\mathcal{H}_{p}^{\lambda}(k, a, c, \alpha, \beta, \mu, \eta)$ and $\mathcal{Q}_{p}^{\lambda}(k, a, c, \alpha, \beta, \mu, \eta)$ which are introduced above. Furthermore, some radius problems are also considered. 


\section{Main Results}

In order to establish our results, we require the following lemmas.

Lemma 1: [6] Let $u=u_{1}+i u_{2}$ and $v=v_{1}+i v_{2}$ and let $\Psi(u, v)$ be a complex-valued function satisfying the conditions:

1) $\Psi(u, v)$ is continuous in a domain $\mathcal{D} \subset \mathbb{C}^{2}$,

2) $(1,0) \in \mathcal{D}$ and $\Psi(1,0)>0$.

3) $\operatorname{Re} \Psi\left(i u_{2}, v_{1}\right) \leq 0$ whenever $\left(i u_{2}, v_{1}\right) \in \mathcal{D}$ and $v_{1} \leq-\left(1+u_{2}^{2}\right) / 2$.

If $p(z)$ is analytic in $\mathbb{U}$, with $p(0)=1$, such that $\left(p(z), z p^{\prime}(z)\right) \in \mathcal{D}$ and $\operatorname{Re}\left(p(z), z p^{\prime}(z)\right)>0$ for $z \in \mathbb{U}$, then $\operatorname{Re} p(z)>0$.

Lemma 2: [12] If $h(z)$ is analytic in $\mathbb{U}$ with $p(0)=1$, and if $\eta$ is a complex number satisfying $\operatorname{Re} \eta \geq 0(\eta \neq 0)$, then

$\operatorname{Re}\left\{h(z)+\eta z h^{\prime}(z)\right\}>\alpha(0 \leq \alpha<1)$ implies

$$
\operatorname{Re} h(z)>\alpha(1-\alpha)\left(2 \rho_{1}-1\right) \text {, }
$$

where $\rho_{1}$ is given by

$$
\rho_{1}=\int_{0}^{1} \frac{\mathrm{d} t}{1+t^{\mathrm{Re} \eta}}
$$

which is an increasing function of $\operatorname{Re} \eta$ and $1 / 2 \leq \rho_{1}<1$. The estimate (20) cannot be improved in general.

Lemma 3: [4] Let $q(z)$ be analytic in $\mathbb{U}$ with $q(0)=1$ and $\operatorname{Re} q(z)>0 \quad(z \in \mathbb{U})$. Then, for $|z|=r<1$,

$$
\frac{1-r}{1+r} \leq \operatorname{Re} q(z) \leq|q(z)| \leq \frac{1+r}{1-r}
$$

and

$$
\left|q^{\prime}(z)\right| \leq \frac{2 \operatorname{Re} q(z)}{1-r^{z}} .
$$

We begin by proving the following.

Theorem 1: Let $\eta \geq 0$. If

$$
f \in \mathcal{H}_{p}^{\lambda}(k, a, c, \alpha, \beta, \mu, \eta) \text {, then }
$$

$$
\left(\frac{\mathcal{I}_{p}^{\lambda}(a+1, c) f(z)}{\mathcal{I}_{p}^{\lambda}(a+1, c) g(z)}\right)^{\mu} \in \mathcal{P}_{k}(\gamma),
$$

where

$$
\gamma=\frac{2 a \alpha \mu+\eta \delta}{2 a \mu+\eta \delta}
$$

and $g \in \mathcal{A}(p)$ satisfies the condition (16) and

$$
\delta=\frac{\operatorname{Re} h_{0}(z)}{\left|h_{0}(z)\right|^{2}}, \quad h_{0}(z)=\frac{\mathcal{I}_{p}^{\lambda}(a, c) g(z)}{\mathcal{I}_{p}^{\lambda}(a+1, c) g(z)} .
$$

Proof. Let $f \in \mathcal{H}_{p}^{\lambda}(k, a, c, \alpha, \beta, \mu, \eta)$ and set

$$
\left(\frac{\mathcal{I}_{p}^{\lambda}(a+1, c) f(z)}{\mathcal{I}_{p}^{\lambda}(a+1, c) g(z)}\right)^{\mu}=(1-\gamma) h(z)+\gamma,
$$

where $h(z)$ is analytic in $\mathbb{U}$ with $h(0)=1$ and we write

$$
h(z)=\left(\frac{k}{4}+\frac{1}{2}\right) h_{1}(z)-\left(\frac{k}{4}-\frac{1}{2}\right) h_{2}(z) .
$$

A simple computation using (23) and (24) gives

$$
\begin{aligned}
& (1-\eta)\left(\frac{\mathcal{I}_{p}^{\lambda}(a+1, c) f(z)}{\mathcal{I}_{p}^{\lambda}(a+1, c) g(z)}\right)^{\mu} \\
& +\eta\left(\frac{\mathcal{I}_{p}^{\lambda}(a, c) f(z)}{\mathcal{I}_{p}^{\lambda}(a, c) g(z)}\right)\left(\frac{\mathcal{I}_{p}^{\lambda}(a+1, c) f(z)}{\mathcal{I}_{p}^{\lambda}(a+1, c) g(z)}\right)^{\mu-1}-\alpha \\
& =\left(\frac{k}{4}+\frac{1}{2}\right)\left\{(1-\gamma) h_{1}(z)+\gamma-\alpha+\frac{\eta(1-\gamma) z h_{1}^{\prime}(z)}{a \mu h_{0}(z)}\right\} \\
& -\left(\frac{k}{4}-\frac{1}{2}\right)\left\{(1-\gamma) h_{2}(z)+\gamma-\alpha+\frac{\eta(1-\gamma) z h_{2}^{\prime}(z)}{a \mu h_{0}(z)}\right\} .
\end{aligned}
$$

Now we form the functional $\Psi(u, v)$ by choosing $u=h_{i}(z)=u_{1}+i u_{2}$ and $v=z h_{i}^{\prime}(z)=v_{1}+i v_{2}$. Thus

$$
\Psi(u, v)=(1-\gamma) u+\gamma-\alpha+\frac{\eta(1-\gamma) v}{a \mu h_{0}(z)} .
$$

The conditions 1) and 2) of Lemma 1 are clearly satisfied. Therefore, we show that the condition 3) of Lemma 1 is satisfied.

By virtue of (25), we have

$$
\begin{aligned}
\operatorname{Re} \Psi\left(i u_{2}, v_{1}\right) & =\gamma-\alpha+\frac{\eta(1-\gamma) \operatorname{Re} h_{0}(z)}{a \mu\left|h_{0}(z)\right|^{2}} v_{1} \\
& =\gamma-\alpha+\frac{\eta(1-\gamma) \delta}{a \mu} v_{1},
\end{aligned}
$$

where $\delta$ is given by (22). Thus, for $v_{1} \leq-\left(1-u_{2}^{2}\right) / 2$, we obtain

$$
\operatorname{Re} \Psi\left(i u_{2}, v_{1}\right) \leq \gamma-\alpha-\frac{\eta(1-\gamma)\left(1+u_{2}^{2}\right) \delta}{2 a \mu}=\frac{A+B u_{2}^{2}}{2 C}
$$

where 


$$
\begin{gathered}
A=2 a \mu(\gamma-\alpha)-\eta \delta(1-\gamma), \\
B=-\eta \delta(1-\gamma) \text { and } C=a \mu .
\end{gathered}
$$

Since $B \leq 0, C>0$ and $A \leq 0$ by (21), we get $\operatorname{Re} \Psi\left(i u_{2}, v_{1}\right) \leq 0$. Hence, by applying Lemma 1 , it follows that $h_{i} \in \mathcal{P}(i=1,2 ; z \in \mathbb{U})$ which implies that $h \in \mathcal{P}_{k}$. The proof of Theorem 1 is thus completed.

Remark: If we put $a=n+p$ and $c=\lambda=1$ in Theorem 1, we have the result due to Noor and Arif [9, Theorem 3.1].

Theorem 2: Let $\eta \geq 0$. If $f \in \mathcal{Q}_{p}^{\lambda}(k, a, c, \alpha, \beta, \mu, \eta)$, then $\left(\frac{\mathcal{I}_{p}^{\lambda}(a, c) f(z)}{\mathcal{I}_{p}^{\lambda}(a, c) g(z)}\right)^{\mu} \in \mathcal{P}_{k}(\gamma)$, where

$$
\gamma=\frac{2 \alpha \mu(\lambda+p)+\eta \delta}{2 \mu(\lambda+p)+\eta \delta}
$$

and $g \in \mathcal{A}(p)$ satisfies the condition (18) and

$$
\begin{gathered}
\delta=\frac{\operatorname{Re} h_{0}(z)}{\left|h_{0}(z)\right|^{2}}, \\
h_{0}(z)=\frac{\mathcal{I}_{p}^{\lambda+1}(a, c) f(z)}{\mathcal{I}_{p}^{\lambda}(a, c) g(z)} .
\end{gathered}
$$

Proof. Let $f \in \mathcal{Q}_{p}^{\lambda}(k, a, c, \alpha, \beta, \mu, \eta)$ and set

$$
\left(\frac{\mathcal{I}_{p}^{\lambda}(a, c) f(z)}{\mathcal{I}_{p}^{\lambda}(a, c) g(z)}\right)^{\mu}=(1-\gamma) h(z)+\gamma,
$$

where $h(z)$ is analytic in $\mathbb{U}$ with $h(0)=1$. Then, by using same techniques as in the proof of Theorem 1, we obtain the desired result.

We note that $\gamma=\alpha$ when $\beta=0$ in Theorem 1 .

Corollary 1: Let $\eta \geq 1$. If $f \in \mathcal{H}_{p}^{\lambda}(k, a, c, \alpha, 0,1, \eta)$, then

$$
\frac{\mathcal{I}_{p}^{\lambda}(a, c) f(z)}{\mathcal{I}_{p}^{\lambda}(a, c) g(z)} \in \mathcal{P}_{k}(\alpha) \quad(z \in \mathbb{U}) .
$$

Proof. It is clear that, for $\eta \geq 1$,

$$
\begin{aligned}
& \eta \frac{\mathcal{I}_{p}^{\lambda}(a, c) f(z)}{\mathcal{I}_{p}^{\lambda}(a, c) g(z)} \\
& =\left[(1-\eta) \frac{\mathcal{I}_{p}^{\lambda}(a+1, c) f(z)}{\mathcal{I}_{p}^{\lambda}(a+1, c) g(z)}+\eta \frac{\mathcal{I}_{p}^{\lambda}(a, c) f(z)}{\mathcal{I}_{p}^{\lambda}(a, c) g(z)}\right] \\
& \quad+(\eta-1) \frac{\mathcal{I}_{p}^{\lambda}(a+1, c) f(z)}{\mathcal{I}_{p}^{\lambda}(a+1, c) g(z)} .
\end{aligned}
$$

This implies that

$$
\begin{aligned}
& \frac{\mathcal{I}_{p}^{\lambda}(a, c) f(z)}{\mathcal{I}_{p}^{\lambda}(a, c) g(z)} \\
& =\frac{1}{\eta}\left[(1-\eta) \frac{\mathcal{I}_{p}^{\lambda}(a+1, c) f(z)}{\mathcal{I}_{p}^{\lambda}(a+1, c) g(z)}+\eta \frac{\mathcal{I}_{p}^{\lambda}(a, c) f(z)}{\mathcal{I}_{p}^{\lambda}(a, c) g(z)}\right] \\
& \quad+\left(1-\frac{1}{\eta}\right) \frac{\mathcal{I}_{p}^{\lambda}(a+1, c) f(z)}{\mathcal{I}_{p}^{\lambda}(a+1, c) g(z)}=\frac{1}{\eta} P_{1}+\left(1-\frac{1}{\eta}\right) P_{2} .
\end{aligned}
$$

Since $\mathcal{P}_{k}(\alpha)$ is a convex set (see [7]), by using Theorem 1 and Definition 1, we observe that $P_{1}, P_{2} \in \mathcal{P}_{k}(\alpha)$ and

$$
\frac{\mathcal{I}_{p}^{\lambda}(a, c) f(z)}{\mathcal{I}_{p}^{\lambda}(a, c) g(z)} \in \mathcal{P}_{k}(\alpha),
$$

which completes the proof of Corollary 1.

Making use of Theorem 2 and Definition 2, we can prove the following result.

Corollary 2: Let $\eta \geq 1$. If $f \in \mathcal{Q}_{p}^{\lambda}(k, a, c, \alpha, 0,1, \eta)$, then

$$
\frac{\mathcal{I}_{p}^{\lambda+1}(a, c) f(z)}{\mathcal{I}_{p}^{\lambda+1}(a, c) g(z)} \in \mathcal{P}_{k}(\alpha) \quad(z \in \mathbb{U}) .
$$

Next, by using Lemma 2, we prove the following.

Theorem 3: Let $\eta$ be a complex number satisfying Re $\eta>0$ and let $a>0, c \in \mathbb{R} / \mathbb{Z}_{0}^{-}, \lambda \geq 0$ and $\mu>0$. If $f \in \mathcal{A}(p)$ satisfies the condition

$$
\begin{aligned}
& \left\{(1-\eta)\left(\frac{\mathcal{I}_{p}^{\lambda}(a+1, c) f(z)}{z^{p}}\right)^{\mu}\right. \\
& \left.+\eta \frac{\mathcal{I}_{p}^{\lambda}(a, c) f(z)}{z^{p}}\left(\frac{\mathcal{I}_{p}^{\lambda}(a+1, c) f(z)}{z^{p}}\right)^{\mu-1}\right\} \in \mathcal{P}_{k}(\alpha),
\end{aligned}
$$

then

$$
\left(\frac{\mathcal{I}_{p}^{\lambda}(a+1, c) f(z)}{z^{p}}\right)^{\mu} \in \mathcal{P}_{k}(\sigma) \quad(z \in \mathbb{U}),
$$

where

$$
\sigma=\alpha+(1-\alpha)\left(2 \rho_{1}-1\right) \quad \text { with } \quad \rho_{1}=\int_{0}^{1}\left(1+t^{\frac{\mathrm{Re} \eta}{a \mu}}\right)^{-1} \mathrm{~d} t .
$$

The value of $\sigma$ is best possible and cannot be improved.

Proof. If we set 


$$
\begin{aligned}
& \left(\frac{\mathcal{I}_{p}^{\lambda}(a+1, c) f(z)}{z^{p}}\right)^{\mu} \\
& =h(z)=\left(\frac{k}{4}+\frac{1}{2}\right) h_{1}(z)-\left(\frac{k}{4}-\frac{1}{2}\right) h_{2}(z),
\end{aligned}
$$

then $h(0)=1$ and $h$ is analytic in $\mathbb{U}$. By applying (7), we have

$$
\begin{aligned}
& (1-\eta)\left(\frac{\mathcal{I}_{p}^{\lambda}(a+1, c) f(z)}{z^{p}}\right)^{\mu} \\
& +\eta \frac{\mathcal{I}_{p}^{\lambda}(a, c) f(z)}{z^{p}}\left(\frac{\mathcal{I}_{p}^{\lambda}(a+1, c) f(z)}{z^{p}}\right)^{\mu-1} \\
& =h(z)+\frac{\eta}{a \mu} z h^{\prime}(z) \in \mathcal{P}_{k}(\alpha) .
\end{aligned}
$$

Therefore, by virtue of Lemma 2, we see that $h_{i} \in \mathcal{P}(\sigma)(i=1,2)$, where $\sigma$ is given by (26). Hence we conclude that $h \in \mathcal{P}_{k}(\alpha)$, which evidently proves Theorem 3.

By using (8) instead of (7) in Theorem 3, we have the following.

Theorem 4: Let $\eta$ be a complex number satisfying $\operatorname{Re} \eta>0$ and let $a, c \in \mathbb{R} / \mathbb{Z}_{0}^{-}, \lambda \geq 0$ and $\mu>0$. If $f \in \mathcal{A}(p)$ satisfies the condition

$$
\begin{aligned}
& \left\{(1-\eta)\left(\frac{\mathcal{I}_{p}^{\lambda}(a+1, c) f(z)}{z^{p}}\right)^{\mu}\right. \\
& \left.+\eta \frac{\mathcal{I}_{p}^{\lambda+1}(a, c) f(z)}{z^{p}}\left(\frac{\mathcal{I}_{p}^{\lambda}(a, c) f(z)}{z^{p}}\right)^{\mu-1}\right\} \in \mathcal{P}_{k}(\alpha),
\end{aligned}
$$

then

$$
\left(\frac{\mathcal{I}_{p}^{\lambda}(a, c) f(z)}{z^{p}}\right)^{\mu} \in \mathcal{P}_{k}(\sigma) \quad(z \in \mathbb{U}),
$$

where $\sigma$ is given by (26) with

$\rho_{1}=\int_{0}^{1}\left(1+t^{\frac{\mathrm{Re} \eta}{\mu(\lambda+p)}}\right)^{-1} \mathrm{~d} t$ The value of $\sigma$ is best possible and cannot be improved.

Theorem 5. Let $0 \leq \eta_{2}<\eta_{1}$. Then $\mathcal{H}_{p}^{\lambda}\left(k, a, c, \alpha, 0, \mu, \eta_{1}\right) \subset \mathcal{H}_{p}^{\lambda}\left(k, a, c, \alpha, 0, \mu, \eta_{2}\right)$.

Proof. If $\eta_{2}=0$, then the proof is immediate from Theorem 1. Let $\eta_{2}>0$ and $f \in \mathcal{H}_{p}^{\lambda}\left(k, a, c, \alpha, 0, \mu, \eta_{1}\right)$. Then there exist two functions $H_{1}, H_{2} \in \mathcal{P}_{k}(\alpha)$ such that

$$
\begin{aligned}
& \left(1-\eta_{1}\right)\left(\frac{\mathcal{I}_{p}^{\lambda}(a+1, c) f(z)}{\mathcal{I}_{p}^{\lambda}(a+1, c) g(z)}\right)^{\mu} \\
& +\eta_{1}\left(\frac{\mathcal{I}_{p}^{\lambda}(a, c) f(z)}{\mathcal{I}_{p}^{\lambda}(a, c) g(z)}\right)\left(\frac{\mathcal{I}_{p}^{\lambda}(a+1, c) f(z)}{\mathcal{I}_{p}^{\lambda}(a+1, c) g(z)}\right)^{\mu-1}=H_{1}(z)
\end{aligned}
$$

and

$$
\left(\frac{\mathcal{I}_{p}^{\lambda}(a+1, c) f(z)}{\mathcal{I}_{p}^{\lambda}(a+1, c) g(z)}\right)^{\mu}=H_{2}(z)
$$

Then

$$
\begin{aligned}
& \left(1-\eta_{2}\right)\left(\frac{\mathcal{I}_{p}^{\lambda}(a+1, c) f(z)}{\mathcal{I}_{p}^{\lambda}(a+1, c) g(z)}\right)^{\mu} \\
& +\eta_{2}\left(\frac{\mathcal{I}_{p}^{\lambda}(a, c) f(z)}{\mathcal{I}_{p}^{\lambda}(a, c) g(z)}\right)\left(\frac{\mathcal{I}_{p}^{\lambda}(a+1, c) f(z)}{\mathcal{I}_{p}^{\lambda}(a+1, c) g(z)}\right)^{\mu-1} \\
& =\frac{\eta_{2}}{\eta_{1}} H_{1}(z)+\left(1-\frac{\eta_{2}}{\eta_{1}}\right) H_{2}(z) .
\end{aligned}
$$

Since $\mathcal{P}_{k}(\alpha)$ is convex set (see [7]), it follows that the right hand side of (2.8) belongs to $\mathcal{P}_{k}(\alpha)$, which proves Theorem 5 .

Next, we consider the generalized Bernardi-LiberaLivingston integral operator $\mathcal{J}_{v}(v>-p)$ defined by (cf. $[1,8]$, and [15])

$$
\mathcal{J}_{v}(f)(z)=\frac{v+p}{z^{v}} \int_{0}^{z} t^{v-1} f(t) \mathrm{d} t \quad(f \in \mathcal{A}(p) ; v>-p) .
$$

Theorem 6: Let $\eta$ be a complex number satisfying Re $\eta>0$ and let $f(z) \in \mathcal{A}(p)$ and $\mathcal{J}_{v}(f)$ be given by (2.9). If

$$
\left\{(1-\eta) \frac{\mathcal{I}_{p}^{\lambda}(a, c) \mathcal{J}_{v}(f)(z)}{z^{p}}+\eta \frac{\mathcal{I}_{p}^{\lambda}(a, c) f(z)}{z^{p}}\right\} \in \mathcal{P}_{k}(\alpha)
$$

then

$$
\frac{\mathcal{I}_{p}^{\lambda}(a, c) \mathcal{J}_{v}(f)(z)}{z^{p}} \in \mathcal{P}_{k}(\alpha),
$$

where $\sigma$ is given by (26) with

$$
\rho_{1}=\int_{0}^{z}\left(1+t^{\frac{\operatorname{Re} \eta}{p+v}}\right)^{-1} \mathrm{~d} t
$$

Proof. From (28), we obtain

$$
\begin{aligned}
& z\left(\mathcal{I}_{p}^{\lambda}(a, c) \mathcal{J}_{v}(f)(z)\right)^{\prime} \\
& =(p+v) \mathcal{I}_{p}^{\lambda}(a, c)(f)(z)-v \mathcal{I}_{p}^{\lambda}(a, c) \mathcal{J}_{v}(f)(z) .
\end{aligned}
$$


Let $\frac{\mathcal{I}_{p}^{\lambda}(a, c) \mathcal{J}_{v}(f)(z)}{z^{p}}=h(z)$. Then, by virtue of (29), we have

$$
\begin{aligned}
& (1-\eta) \frac{\mathcal{I}_{p}^{\lambda}(a, c) \mathcal{J}_{v}(f)(z)}{z^{p}}+\eta \frac{\mathcal{I}_{p}^{\lambda}(a, c) f(z)}{z^{p}} \\
& =h(z)+\frac{\eta z h^{\prime}(z)}{p+v} \in \mathcal{P}_{k}(\alpha) .
\end{aligned}
$$

Hence, by using Lemma 2, we obtain the desired result.

Finally, we consider the converse case of Theorem 1 as follows.

Theorem 7: Let $f \in \mathcal{H}_{p}^{\lambda}(k, a, c, \alpha, \beta, \mu, 0)$. Then $f \in \mathcal{H}_{p}^{\lambda}(k, a, c, \alpha, \beta, \mu, \eta)$ for $|z|<R$, where $R$ is given by

$$
R=\frac{a \mu}{a \mu(1-\beta)+\eta+\sqrt{(a \beta \mu)^{2}+\eta^{2}+2 a \eta \mu(1-\beta)}} .
$$

Proof. Let

$$
H(z)=\left(\frac{\mathcal{I}_{p}^{\lambda}(a+1, c) f(z)}{\mathcal{I}_{p}^{\lambda}(a+1, c) g(z)}\right)^{\mu}
$$

and

$$
H_{0}(z)=\frac{\mathcal{I}_{p}^{\lambda}(a, c) g(z)}{\mathcal{I}_{p}^{\lambda}(a+1, c) g(z)}
$$

and since $f \in \mathcal{H}_{p}^{\lambda}(k, a, c, \alpha, 0, \mu, 0)$, it follows that $H \in \mathcal{P}_{k}(\alpha)$ and $H_{0} \in \mathcal{P}_{k}(\alpha)$. Proceeding as in Theorem 1, for

$H(z)=(1-\alpha) h(z)+\alpha$ and $H_{0}(z)=(1-\beta) h(z)+\beta$ with $h \in \mathcal{P}_{k}, h_{0} \in \mathcal{P}$, we obtain

$$
\begin{aligned}
& \frac{1}{1-\alpha}\left\{(1-\eta)\left(\frac{\mathcal{I}_{p}^{\lambda}(a+1, c) f(z)}{\mathcal{I}_{p}^{\lambda}(a+1, c) g(z)}\right)^{\mu}\right. \\
& \left.+\eta_{2}\left(\frac{\mathcal{I}_{p}^{\lambda}(a, c) f(z)}{\mathcal{I}_{p}^{\lambda}(a, c) g(z)}\right)\left(\frac{\mathcal{I}_{p}^{\lambda}(a+1, c) f(z)}{\mathcal{I}_{p}^{\lambda}(a+1, c) g(z)}\right)^{\mu-1}-\alpha\right\} V_{d}^{\prime} \\
& =h(z)+\frac{\eta}{a \mu} \frac{z h^{\prime}(z)}{(1-\beta) h_{0}+\beta} \\
& =\left(\frac{k}{4}+\frac{1}{2}\right)\left[h_{1}(z)+\frac{\eta}{a \mu} \frac{z h_{1}^{\prime}(z)}{(1-\beta) h_{0}+\beta}\right] \\
& -\left(\frac{k}{4}-\frac{1}{2}\right)\left[h_{2}(z)+\frac{\eta}{a \mu} \frac{z h_{2}^{\prime}(z)}{(1-\beta) h_{0}+\beta}\right]
\end{aligned}
$$

By applying Lemma 3 , for $h_{i} \in \mathcal{P}(i=0,1,2)$ and $|z|=r<1$, we have

$$
\begin{aligned}
& \operatorname{Re}\left[h_{i}(z)+\frac{\eta}{a \mu} \frac{z h_{i}^{\prime}(z)}{(1-\beta) h_{0}+\beta}\right] \\
& \geq \operatorname{Re} h_{i}(z)\left[1-\frac{\eta}{a \mu} \frac{2 r}{1-r^{2}}\left(\frac{1+r}{1-(1-2 \beta) r}\right)\right] \\
& \geq \operatorname{Re} h_{i}(z)\left[1-\frac{2 \eta r}{a \mu(1-r)\{1-(1-2 \beta) r\}}\right] \\
& \geq \operatorname{Re} h_{i}(z)\left[\frac{(1-2 \beta) a \mu r^{2}-2\{(1-\beta) a \mu+\eta\} r+a \mu}{a \mu(1-r)\{1-(1-2 \beta) r\}}\right] .
\end{aligned}
$$

Hence, the hand right side of (31) is positive for $|z|=r<R$, where $R$ is given by (30). This completes the proof of Theorem 7 .

Theorem 8. Let $f \in \mathcal{Q}_{p}^{\lambda}(k, a, c, \alpha, \beta, \mu, 0)$. Then $f \in \mathcal{Q}_{p}^{\lambda}(k, a, c, \alpha, \beta, \mu, \eta)$ for $|z|<R$, where $R$ is given by

$$
\begin{aligned}
& R=\mu(\lambda+p)(\mu(1-\beta)(\lambda+p)+\eta \\
& \left.+\sqrt{[\beta \mu(\lambda+p)]^{2}+\eta^{2}+2 \eta \mu(1-\beta)(\lambda+p)}\right)^{-1} .
\end{aligned}
$$

Proof. Let

$$
H(z)=\left(\frac{\mathcal{I}_{p}^{\lambda}(a, c) f(z)}{\mathcal{I}_{p}^{\lambda}(a, c) g(z)}\right)^{\mu}
$$

and

$$
H_{0}(z)=\frac{\mathcal{I}_{p}^{\lambda}(a, c) g(z)}{\mathcal{I}_{p}^{\lambda}(a+1, c) g(z)}
$$

and since $f \in \mathcal{Q}_{p}^{\lambda}(k, a, c, \alpha, 0, \mu, 0)$, it follows that $H \in \mathcal{P}_{k}(\alpha)$ and $H_{0} \in \mathcal{P}(\beta)$. Then, by using same methods as in the proof of Theorem 7 , we obtain the required result.

\section{Acknowledgements}

This work was supported by Daegu National University of Education Research Grant in 2010.

\section{References}

[1] S. D. Bernardi, "Convex and Starlike Univalent Functions," Transactions of the American Mathematical Society, Vol. 135, 1969, pp. 429-446. doi:10.1090/S0002-9947-1969-0232920-2 
[2] B. C. Carlson and D. B. Shaffer, "Starlike and Prestarlike Hypergeometric Functions," SIAM Journal on Mathematical Analysis, Vol. 15, No. 4, 1984, pp. 737-745. doi:10.1137/0515057

[3] N. K. Cho, O. S. Kwon and H. M. Srivastava, "Inclusion Relationships and Argument Properties for Certain Subclasses of Multivalent Functions Associated with a Family of Linear Operator," Journal of Mathematical Analysis and Applications, Vol. 292, No. 2, 2004, pp. 470-483. doi:10.1016/j.jmaa.2003.12.026

[4] A. W. Goodman, "Univalent Functions, Vol. I, II," Polygonal Publishing House, Washington, 1983.

[5] J.-L. Liu and K. I. Noor, "Some Properties of Noor Integral Operator," Journal of Natural Geometry, Vol. 21, 2002, pp. 81-90.

[6] S. S. Miller, "Differential Inequalities and Caratheodory Functions," Bulletin of the American Mathematical Society, Vol. 81, 1975, pp. 78-81. doi:10.1090/S0002-9904-1975-13643-3

[7] K. I. Noor, "On Subclasses of Close-to-Convex Functions of Higher Order," International Journal of Mathematics and Mathematical Sciences, Vol. 15, No. 2, 1992, pp. 279-289. doi:10.1155/S016117129200036X

[8] K. I. Noor, "On New Classes of Integral Operators," Journal of Natural Geometry, Vol. 16, 1999, pp. 71-80.
[9] K. I. Noor and M. Arif, "Generalized Integral Operators Related with $p$-Valent Analytic Functions," Mathematical Inequalities Applications, Vol. 12, No. 1, 2009, pp. 91-98.

[10] J. Patal and N. E. Cho, "Some Classes of Analytic Functions Involving Noor Integral Operator," Journal of Mathematical Analysis and Applications, Vol. 312, No. 2, 2005, pp. 564-575. doi:10.1016/j.jmaa.2005.03.047

[11] B. Pinchuk, "Functions with Bounded Boundary Rotation," Israel Journal of Mathematics, Vol. 10, No. 1, 1971, pp. 7-16. doi:10.1007/BF02771515

[12] S. Ponnusamy, "Differential Subordination and Bazilevic Functions," Proceedings Mathematical Sciences, Vol. 105, No. 2, 1995, pp. 169-186. doi:10.1007/BF02880363

[13] H. Saitoh, "A Linear Operator and Its Applications of First Order Differential Subordinations," Mathematica Japonica, Vol. 44, 1996, pp. 31-38.

[14] J. Sokół and L. Trojnar-Spelina, "Convolution Properties for Certain Classes of Multivalent Functions," Journal of Mathematical Analysis and Applications, Vol. 337, No. 2, 2008, pp. 1190-1197. doi:10.1016/i.jmaa.2007.04.055

[15] H. M. Srivastava and S. Owa (Eds.), "Current Topics in analytic Function Theory," World Scientific, Singapore, 1992. 